\title{
Spectroscopic behaviour of the unusual Ae star HD 190073 ${ }^{\star}$
}

\author{
M. A. Pogodin ${ }^{1,2}$, G. A. P. Franco ${ }^{3}$, and D. F. Lopes ${ }^{4}$ \\ 1 Pulkovo Observatory, Saint-Petersburg, 196140, Russia \\ e-mail: pogodin@gao.spb.ru \\ 2 Isaac Newton Institute of Chile, Saint-Petersburg Branch, Russia \\ 3 Departamento de Física - UFMG, Belo Horizonte, Brazil \\ e-mail: franco@fisica.ufmg.br \\ ${ }^{4}$ Observatório Nacional, Rio de Janeiro, Brazil \\ e-mail: dalton@on.br
}

Received 5 September 2003 / Accepted 31 March 2005

\begin{abstract}
The results of high-resolution spectroscopy of the peculiar Ae star HD 190073 obtained within the framework of a cooperative observing programme in 1994-2002 are presented. The temporal behaviour of the $\mathrm{H} \alpha, \mathrm{H} \beta, \mathrm{H} \gamma, \mathrm{H} \delta, \mathrm{He} \mathrm{I} \lambda 5876 \AA$, $\mathrm{NaID}$ and other circumstellar line profiles are investigated in detail. Special attention has been paid to the analysis of the deep multicomponent blueshifted $\mathrm{Ca}$ II $\mathrm{H}$ and $\mathrm{K}$ absorption lines. It has been found that the fine structure of their profiles is variable on timescales from months to decades. The analysis of the circumstellar spectrum of HD 190073, rich in shell-like and emission lines with narrow absorption cores, allows us to conclude that all absorption lines and cores are likely to be of photospheric origin. The emission lines are variable in time and demonstrate signs of a stellar wind as well as a dense equatorial circumstellar disk. As a preliminary hypothesis, we propose that a global magnetic field of a specific topology can be responsible for the formation of stable latitudinal stratification of the outflowing gas resulting in appearance of the complex structure of the $\mathrm{Ca}$ II $\mathrm{H}$ and $\mathrm{K}$ line profiles. We emphasise that a measurement of the stellar magnetic field and an investigation of its detailed configuration would be an important step in understanding the nature of HD 190073.
\end{abstract}

Key words. techniques: spectroscopic - stars: circumstellar matter - stars: emission-line, Be - stars: individual: HD 190073 stars: magnetic field - stars: pre-main sequence

\section{Introduction}

HD 190073 (MWC 325, A2 IIIe - B9 IVep+sh) is a very remarkable early-type object with emission lines in the visible spectrum. Over a long period of study from the early 1930s up to now, HD 190073 was variously classified as a peculiar Bep star (Allen \& Swings 1976) or as an evolved postmain sequence A giant (Cuttela \& Ringuelet 1990). Recently HD 190073 has gained recognition as a young Herbig Ae/Be star (Cidale et al. 2000; de Winter et al. 2001; Mora et al. 2001). In spite of being situated in the constellation of Aquila rather far from well-known regions of star formation, it displays a large far-IR excess due to thermal radiation of cool circumstellar (CS) dust (Sitko 1981). The energy distribution of HD 190073 in this spectral region is similar to that of well known Herbig Ae stars, like AB Aur, HD 163296 and HD 31648 (Malfait et al. 1998). According to Chen et al. (2000), the size and the structure of the $9.7 \mu \mathrm{m}$ silicate feature in the spectrum of the object resembles those observed in AB Aur and HD 163296.

* Based on observations collected at the Crimean Astrophysical Observatory (CrAO, Ukraine), Observatório do Pico dos Dias (LNA, Brazil), and European Southern Observatory (ESO/La Silla, Chile).
Studies in other spectral regions confirm the similarity of HD 190073 to already recognized Herbig Ae/Be stars (HAEBEs). In particular, the IUE UV low-resolution spectra of the object contain the resonance $\mathrm{Mg}$ II doublet with a distinctive P Cyg-type profile, several emission lines of O I, O II, Si II, Si III, Si IV, and C IV as well as absorption Fe II lines, thus resembling the UV spectra of AB Aur, HD 163296 and HD 31648 (Sitko et al. 1981; Imhoff 1994; Grady et al. 1996).

Despite these similarities, there are several distinctions between HD 190073 and other HAEBEs of the same type. Table 1 presents some observational parameters of HD 190073 in comparison with those for several well known Herbig Ae stars. As one can see, HD 190073 is more distant and, consequently, more luminous than AB Aur, HD 163296 and HD 31648. The second distinctive property of HD 190073 is its very small value of projected rotational velocity $v \sin i$.

One of the first detailed descriptions of the emission spectrum of HD 190073 was given by Merrill (1933). He reported the presence of bright hydrogen, metallic and $D_{1,2}$ sodium lines, and noted a complex structure of the $\mathrm{Ca}$ II $\mathrm{H}, \mathrm{K}$ absorption profiles. This structure is the most conspicuous feature of the object's spectrum, and makes HD 190073 one of the most puzzling emission early-type stars. The line profile of the 
Table 1. General observational parameters of HD 190073 in comparison with those for several HAEBEs of the same type.

\begin{tabular}{cccccc}
\hline \hline Object & AB Aur & HD 163296 & HD 31648 & HD 190073 & References \\
\hline $\log T_{\text {eff }}(\mathrm{K})$ & 3.98 & 3.97 & 3.94 & 3.95 & 1 \\
$V(\mathrm{mag})$ & 7.1 & 6.9 & 7.7 & 7.8 & 1 \\
$A_{\mathrm{v}}(\mathrm{mag})$ & 0.50 & 0.25 & 0.25 & 0.19 & 1 \\
$\pi(\mathrm{mas})$ & $6.9 \pm 1.0$ & $8.2 \pm 1.0$ & $7.6 \pm 1.2$ & $0.2 \pm 1.1$ & 1 \\
$r(\mathrm{pc})$ & $144 \pm 20$ & $122 \pm 15$ & $131 \pm 20$ & $\geq 290$ & 1 \\
$v \sin i\left(\mathrm{~km} \mathrm{~s}^{-1}\right)$ & $80 \pm 5$ & $120 \pm 30$ & $85 \pm 5$ & $15 \pm 5$ & $1,2,3$ \\
\hline
\end{tabular}

(1) - van den Ancker et al. (1998);

(2) - Corporon \& Lagrange (1999);

(3) - Glagolevskij \& Chountonov (1998).

doublet consists of a number of blueshifted absorption components of different width and depth. All the components are variable in intensity but stable in their velocity positions which have remained constant during several decades. Three of the strongest features stand out: the first one corresponds to the rest wavelength; the second deep absorption is centered at $-180 \mathrm{~km} \mathrm{~s}^{-1}$ and a wide very deep complex blend is found in the range from -320 to $-290 \mathrm{~km} \mathrm{~s}^{-1}$. Variations in the $\mathrm{H}$ and $\mathrm{K}$ absorption features were established by Swings \& Struve (1940) and Struve \& Swings (1942). Scargle (1973) stated that the radial velocities of the higher-velocity system are related to the velocities of the components in the second group by a $2: 1$ ratio. He proposed a pure scattering model in order to explain the ejection of $\mathrm{Ca}^{+}$ions from the atmosphere of the star, a scenario that has been developed by Tifft (1977). However, Surdej \& Swings (1976a) concluded that such a ratio did not appear to be significant. Only part of the features corresponds to the ratio and these absorptions are not the strongest among other blueshifted components of the profile. Surdej \& Swings (1976a) analyzed a 24 spectra sample of HD 190073 obtained from 1943 to 1974 and found a correlation between the structure of the $\mathrm{Ca}$ II H, K profiles and the P Cyg absorption in the profiles of Balmer lines. They determined that a fusion between two Ca II components at -180 and $-300 \mathrm{~km} \mathrm{~s}^{-1}$ tended to appear and the first of them became stronger than the second one when the blueshifted absorption component of the Balmer line profiles was strong and wide. They explained these correlations in terms of a model based on the selective effect of radiative force (Surdej \& Swings 1976b, 1977).

The magnetic field of HD 190073 has been measured by Babcock (1958); his measurements yielded different intensity and polarity for different elements and ions: $\sim-270 \mathrm{G}$ for the $\mathrm{Ca}$ II $\mathrm{H}$ and $\mathrm{K}$ components, $\sim 0 \mathrm{G}$ for lines of ionized metals and $\sim+270 \mathrm{G}$ for lines of neutral metals. Later magnetometric observations of Glagolevskij \& Chountonov (1998) did not confirm the existence of a significant magnetic field in HD 190073, but their method allowed them to determine only an average value of the field on the basis of measurement of a large number of visual spectral lines. It would be important to have their measurements repeated separately for lines of different elements and ions.
Multi-color WLUBV-photometry of HD 190073 showed that the object was variable on a timescale of several days but displayed no periodic change of brightness (van Genderen 1971; Deul \& van Genderen 1983).

Taking into account that the star may possess a global magnetic field and displays a strong stellar wind revealed by the P Cyg structure of different spectral lines, Cuttela \& Ringuelet (1990) proposed a model for HD 190073 which takes into account an expanding spherical chromosphere and is based on the MHD model of Low \& Tsinganos (1986). Additionally, this model makes use of some results from calculations conducted by Lamers \& Waters (1984) and Catala \& Kunasz (1987).

As one can see in this overview, HD 190073 demonstrates many properties resembling those observed in "classical" HAEBEs, but it displays some peculiarities; making the object unusual among Herbig B8e - A2e stars.

The main purposes of our paper are: a) to make a new contribution towards a better understanding of the peculiar spectrum of this star on the basis of our new high-resolution spectroscopic data; and b) to compare the spectroscopic behaviour of HD 190073 with that of well-known Herbig Ae stars of similar type.

\section{Observations}

The high-resolution spectroscopic observations of HD 190073 in spectral bands covering the $\mathrm{H} \alpha, \mathrm{H} \beta, \mathrm{He} \mathrm{I} \lambda 5876 \AA$ and Na I D lines were collected between June 1998 and March 2002 at the Crimean Astrophysical Observatory $(\mathrm{CrAO})$ of Ukraine and at the Observatório do Pico dos Dias (LNA/MCT) of Brazil.

At the CrAO the $2.6 \mathrm{~m}$ Shajn telescope equipped with a CCD detector placed in the first camera of the coudéspectrograph was used yielding a resolving power $R \sim 30000$. The detector SDS-9000 "Photometric GmbH" provided a wavelength coverage $(\Delta \lambda)$ of about $60 \AA$. Data reduction followed standard procedures and was done with the SPE computer code developed by S. G. Sergeev.

The observations collected at LNA were carried out using the coudé-spectrograph installed at the $1.6 \mathrm{~m}$ telescope and equipped with a $770 \times 1152$ pixels CCD detector. A spectral resolving power $R$ of 15000 was achieved in each spectrum 
Table 2. Collection of HD 190073 spectra obtained at CrAO and LNA.

\begin{tabular}{|c|c|c|c|c|c|}
\hline Date & $\begin{array}{c}\text { JD } \\
2400000+\end{array}$ & Lines & $N$ & $\begin{array}{c}S / N \text { at } \\
\text { the } F_{\mathrm{c}} \text { level }\end{array}$ & Observatory \\
\hline \multirow[t]{5}{*}{16.06 .98} & 50980.64 & $\mathrm{H} \alpha$ & 1 & 110 & LNA \\
\hline & 50980.67 & $\mathrm{He} \mathrm{I}+\mathrm{Na} \mathrm{I}$ & 1 & 150 & \\
\hline & 50980.69 & $\mathrm{H} \beta$ & 1 & 100 & \\
\hline & 50980.72 & $\mathrm{H} \alpha$ & 1 & 110 & \\
\hline & 50980.74 & $\mathrm{He} \mathrm{I}+\mathrm{Na} \mathrm{I}$ & 1 & 180 & \\
\hline \multirow[t]{2}{*}{04.08 .98} & 51029.69 & $\mathrm{He} \mathbf{I}+\mathrm{Na} \mathbf{I}$ & 1 & 170 & LNA \\
\hline & 51029.71 & $\mathrm{H} \alpha$ & 1 & 80 & \\
\hline 05.08 .98 & 51030.67 & $\mathrm{H} \alpha$ & 1 & 60 & LNA \\
\hline \multirow[t]{2}{*}{24.03 .99} & 51261.59 & $\mathrm{He} \mathrm{I}+\mathrm{Na} \mathrm{I}$ & 1 & 130 & $\mathrm{CrAO}$ \\
\hline & 51261.60 & $\mathrm{H} \alpha$ & 1 & 75 & \\
\hline \multirow[t]{2}{*}{28.03 .99} & 51265.59 & $\mathrm{He} \mathrm{I}+\mathrm{Na} \mathbf{I}$ & 1 & 90 & $\mathrm{CrAO}$ \\
\hline & 51265.61 & $\mathrm{H} \alpha$ & 1 & 50 & \\
\hline \multirow[t]{2}{*}{31.03 .99} & 51268.54 & $\mathrm{He} \mathrm{I}+\mathrm{Na} \mathrm{I}$ & 1 & 110 & $\mathrm{CrAO}$ \\
\hline & 51268.56 & $\mathrm{H} \alpha$ & 1 & 65 & \\
\hline 25.09 .99 & 51445.30 & $\mathrm{H} \alpha$ & 1 & 100 & LNA \\
\hline \multirow[t]{2}{*}{12.05 .00} & 51676.80 & $\mathrm{H} \alpha$ & 1 & 110 & LNA \\
\hline & 51676.83 & $\mathrm{He} \mathrm{I}+\mathrm{Na} \mathrm{I}$ & 1 & 170 & \\
\hline \multirow[t]{2}{*}{18.06 .00} & 51713.82 & $\mathrm{He} \mathrm{I}+\mathrm{Na} \mathbf{I}$ & 1 & 180 & LNA \\
\hline & 51713.84 & $\mathrm{H} \alpha$ & 1 & 100 & \\
\hline \multirow[t]{3}{*}{09.08 .00} & 51765.53 & $\mathrm{He} \mathrm{I}+\mathrm{Na} \mathrm{I}$ & 1 & 150 & LNA \\
\hline & 51765.57 & $\mathrm{H} \beta$ & 1 & 80 & \\
\hline & 51765.68 & $\mathrm{H} \alpha$ & 1 & 100 & \\
\hline \multirow[t]{3}{*}{26.10 .01} & 52209.21 & $\mathrm{He} \mathrm{I}+\mathrm{Na} \mathbf{I}$ & 1 & 75 & $\mathrm{CrAO}$ \\
\hline & 52209.30 & $\mathrm{H} \beta$ & 1 & 50 & \\
\hline & 52209.31 & $\mathrm{H} \alpha$ & 12 & $45-50$ & \\
\hline \multirow[t]{3}{*}{27.10 .01} & 52210.20 & $\mathrm{He} \mathbf{I}+\mathrm{Na} \mathbf{I}$ & 1 & 80 & $\mathrm{CrAO}$ \\
\hline & 52210.30 & $\mathrm{H} \alpha$ & 12 & $35-60$ & \\
\hline & 52210.31 & $\mathrm{H} \beta$ & 1 & 30 & \\
\hline 29.10 .01 & 52212.11 & $\mathrm{H} \alpha$ & 1 & 100 & $\mathrm{CrAO}$ \\
\hline \multirow[t]{2}{*}{05.03 .02} & 52339.63 & $\mathrm{He} \mathrm{I}+\mathrm{Na} \mathrm{I}$ & 1 & 45 & $\mathrm{CrAO}$ \\
\hline & 52339.65 & $\mathrm{H} \alpha$ & 1 & 120 & \\
\hline
\end{tabular}

The observing date is presented in the form DD.MM.YY; the heliocentric Julian date (JD) is given either for the middle point of the exposure or the whole observing time during the night (in case of multiple observations); $N$ is the number of spectra obtained during the night; the signal-to-noise ratio $(S / N)$ at the continuum level $F_{\mathrm{c}}$ and the observatory where the spectra were obtained are indicated in the last two columns.

with $\Delta \lambda$ of about $150 \AA$. The reduction process also followed the standard procedure and was done with the help of the IRAF packages. Table 2 presents the observing data obtained at $\mathrm{CrAO}$ and LNA.

These data have been complemented by several highresolution échelle spectra $(R=48000)$ covering the wide spectral region from $3230 \AA$ to $9200 \AA$ A obtained in 1999-2000 at the European Southern Observatory (ESO, Chile) with the FEROS-spectrograph mounted at the $1.5 \mathrm{~m}$ telescope.

Additionally, we used in our investigation the échelle spectra of HD $190073(\lambda \lambda 3900-6800 \AA, R=42000)$ obtained in 1994-1997 by P. Corporon (Corporon \& Lagrange 1999) at the Observatoire de Haute-Provence (OHP, France) with the ÉLODIE-spectrograph installed at the $1.93 \mathrm{~m}$ telescope. 
Table 3. Summary of échelle spectra of HD 190073 obtained at OHP (Corporon \& Lagrange 1999) and ESO.

\begin{tabular}{|c|c|c|c|c|c|c|c|}
\hline \multirow[t]{2}{*}{ Date } & \multirow{2}{*}{$\begin{array}{c}\text { JD } \\
2400000+\end{array}$} & \multirow{2}{*}{$\begin{array}{c}\text { Spectral } \\
\text { region }(\AA)\end{array}$} & \multicolumn{4}{|c|}{$S / N$ at given $\lambda(\AA)$} & \multirow[t]{2}{*}{ Observatory } \\
\hline & & & 3900 & 4500 & 5500 & 6500 & \\
\hline 27.08 .94 & 49591.42 & $3900-6800$ & 30 & 70 & 85 & 80 & OHP \\
\hline 16.11 .94 & 49673.26 & $3900-6800$ & 25 & 50 & 70 & 65 & \\
\hline 19.08 .95 & 49948.40 & $3900-6800$ & 25 & 55 & 75 & 70 & \\
\hline 14.03 .96 & 50155.68 & $3900-6800$ & 40 & 80 & 115 & 110 & \\
\hline 28.07 .96 & 50292.49 & $3900-6800$ & 20 & 40 & 50 & 50 & \\
\hline 06.07 .97 & 50636.48 & $3900-6800$ & 40 & 100 & 140 & 130 & \\
\hline 23.06 .99 & 51352.36 & $3230-9200$ & 40 & 85 & 100 & 80 & ESO \\
\hline 28.02 .00 & 51602.41 & $3230-9200$ & 20 & 75 & 60 & 40 & \\
\hline 22.04 .00 & 51656.33 & $3230-9200$ & 60 & 140 & 160 & 130 & \\
\hline 16.06 .00 & 51711.43 & $3230-9200$ & 40 & 80 & 120 & 100 & \\
\hline
\end{tabular}

The same as for Table 2 but the $(S / N)$ ratio at the continuum level $F_{\mathrm{c}}$ is given separately for four narrow spectral bands centered at different wavelengths.

The échelle spectra allowed us to separate extracts in different spectral regions containing lines of our interest: $\mathrm{Ca}$ II $\mathrm{H}$ and $\mathrm{K}$, $\mathrm{H} \gamma, \mathrm{H} \delta, \mathrm{Mg}$ II, Fe II (42), etc. The échelle spectra of HD 190073 are collected in Table 3 .

The optical data were supplemented by UV data retrieved from the INES database. Three high dispersion spectra of HD 190073 have been recorded with IUE and the long wavelength camera in the spectral range 2000-3200 $\AA$. These spectra were collected on October 11, 1980 (LWR08996), November 14, 1981 (LWR11977), and June 18, 1993 (LWP25762).

\section{Results}

\subsection{The emission Balmer lines}

Figures 1 and 2 present the central regions of typical profiles of the Balmer lines in the spectrum of HD 190073. The absorption wings of the profiles extend far beyond the boundaries of the figures and are well fitted by synthetic spectra calculated using the photospheric Kurucz' model: $T_{\text {eff }}=9300 \mathrm{~K}, \log g=3.5$, $v \sin i=12 \mathrm{~km} \mathrm{~s}^{-1}$.

We have used numerous photospheric lines for definition of the radial velocity rest frame connected with the star (see Sect. 3.3). It is remarkable that the positions of these features as well as of interstellar (IS) components of the Na ID and the blue Ca II doublets were practically unshifted with respect to the Sun during the whole 8-year period of our observations $\left(+0.3 \pm 0.5 \mathrm{~km} \mathrm{~s}^{-1}-\right.$ for photospheric lines; $-1.5 \pm$ $0.2 \mathrm{~km} \mathrm{~s}^{-1}$ - for NaID IS lines; and $+1.5 \pm 1.0 \mathrm{~km} \mathrm{~s}^{-1}-$ for Ca II IS components).

HD 190073 demonstrates a classical P Cyg II-type profile for $\mathrm{H} \alpha-\mathrm{H} \delta$ lines (according to the classification of Beals 1951). For comparison with the observed $\mathrm{H} \gamma$ and $\mathrm{H} \delta$ lines, synthetic spectra calculated using the above-mentioned photospheric Kurucz' model are superimposed in Fig. 2. Sometimes the blueshifted absorption component disappeared and the profile was transformed into a single emission peak (for example, July 28, 1996; February 28, 2000; October 29, 2001 - respectively the profiles "d", "k" and "p" in Fig. 1). The most

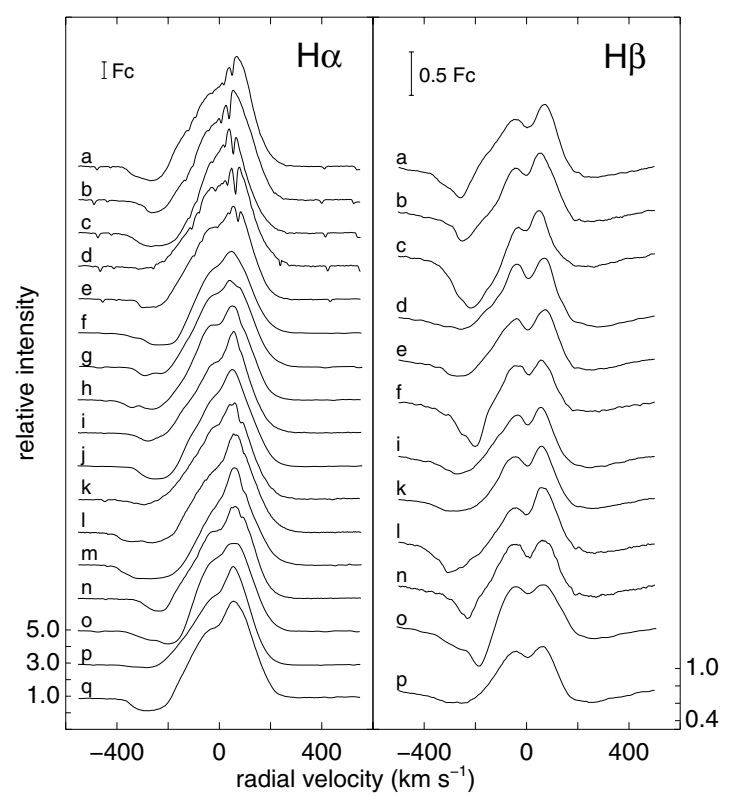

Fig. 1. Normalized profiles of the $\mathrm{H} \alpha$ and $\mathrm{H} \beta$ emission lines observed in the spectrum of HD 190073 on following dates: a) Aug. 27, 1994; b) Nov. 16, 1994; c) Aug. 19, 1995; d) Jul. 28, 1996; e) Jul. 6, 1997; f) June 16, 1998; g) Aug. 4, 1998; h) March 24, 1999; i) June 23, 1999; j) Sept. 25, 1999; k) Feb. 28, 2000; 1) Apr. 22, 2000; m) May 12, 2000; n) June 16, 2000; o) Aug. 9, 2000; p) Oct. 29, 2001; q) March 5, 2002. The velocity scale is given with respect to the star. No attempt has been made to eliminate the water vapour lines overlapping the $\mathrm{H} \alpha$ profiles. The relative intensity scale is given with respect to the lower unshifted spectrum.

developed P Cyg structure of the Balmer line profiles were observed on August 19, 1995; June 16, 1996 and in the spectra obtained from April to August 2000, profiles "c", "f", and from "l" to "o", respectively. It should be noted that such profile transformations are typical for other HAEBEs of B8-A2 spectral classes (Beskrovnaya et al. 1995).

Independently of the presence or absence of the PCygabsorption, all the Balmer line profiles show a notable central 


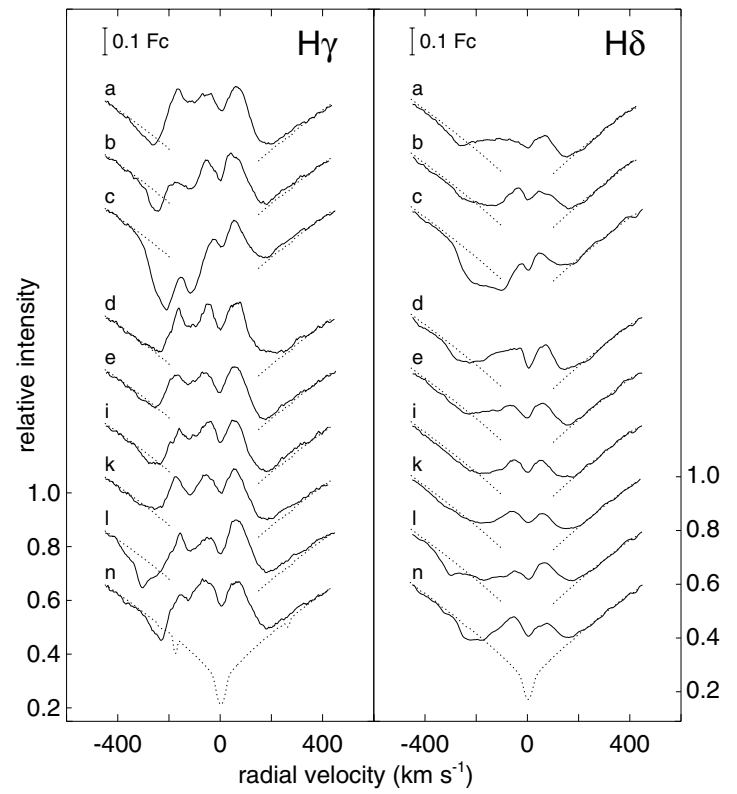

Fig. 2. The same as Fig. 1 but for the $\mathrm{H} \gamma$ and $\mathrm{H} \delta$ lines. Synthetic theoretical photospheric profiles are given by dashed lines (see text for details).

absorption core. In the $\mathrm{H} \alpha$ line profile it looks like a shallow depression. A second blueshifted absorption at $V_{r} \sim-120 \mathrm{~km} \mathrm{~s}^{-1}$ is also seen in the profile of $\mathrm{H} \gamma$. The first absorption is likely to be the central core of the photospheric Balmer line, and the second one is supposingly of CS origin. Is is not clearly seen in other Balmer line profiles. We suppose that it is too shallow to be noticeable in $\mathrm{H} \delta$ and cannot be observed in the $\mathrm{H} \alpha$ and $\mathrm{H} \beta$ profiles because is placed in the region of large intensity gradient. There is no reason to conclude that a nebular narrow emission component is superimposed on the PCyg-type $\mathrm{H} \alpha$ profile like it has been done by Ringuelet \& Sahade (1984). As one can see in Fig. 1 (left panel), the illusion of an additionial narrow component is created on some dates due to the combined effect of a blueshifted depression and a telluric water vapour line seen in the red wing of the emission $\mathrm{H} \alpha$ profile.

The emission Balmer decrement of HD 190073 is unusually gently sloped. The energies radiating in the $\mathrm{H} \alpha, \mathrm{H} \beta$ and $\mathrm{H} \gamma$ lines occur, respectively, in ratio of 1.85:1.00:0.65. This ratio for typical HAEBEs is in the range between 2.50:1.00:0.55 and 3.00:1.00:0.35 (Pogodin 1985).

Investigation of a short-term line profile variability on the basis of $\mathrm{H} \alpha$ data obtained on October 26/27 and 27/28, 2001 shows that no significant variations occurred during that nights in HD 190073. Such variability is usually present in the $\mathrm{H} \alpha$ line originating in the stellar wind of HAEBEs (Pogodin 1994).

\subsection{The He $1 \lambda 5876 \AA$ Aine}

Unlike the majority of the Herbig B8e-A2e stars displaying a complex two-component profile of He I $\lambda 5876 \AA$ line, HD 190073 demonstrates a pure emission profile for this line (Fig. 3) without any signs of the red variable absorption typically observed in spectra of other HAEBEs (Pogodin 1997;

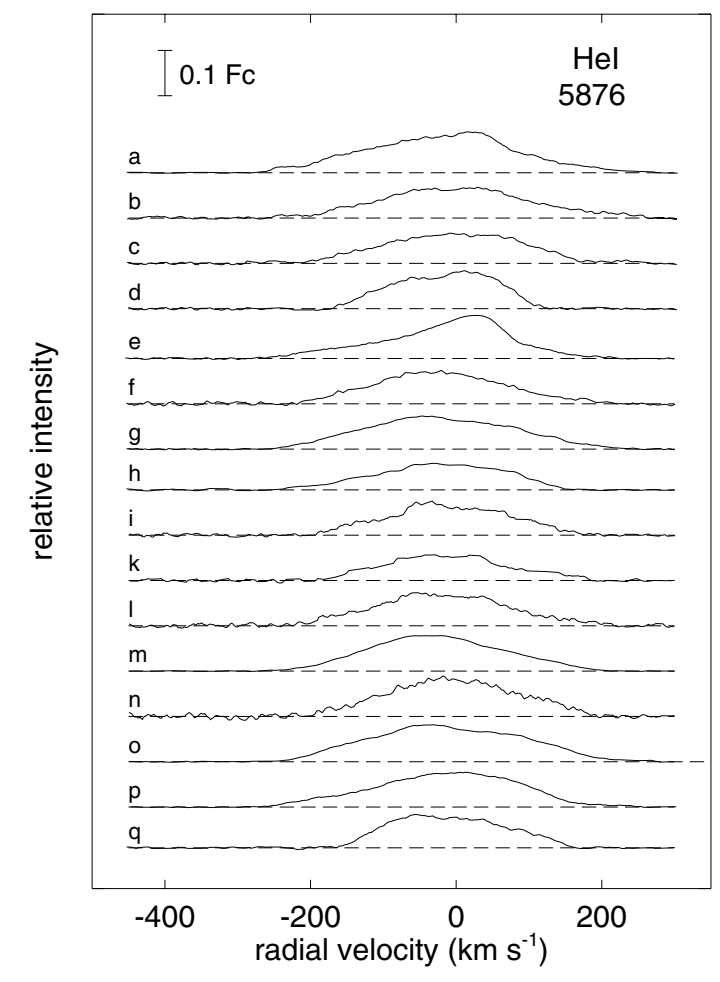

Fig. 3. The same as Fig. 1 but for the emission He I $\lambda 5876 \AA$ line.

Beskrovnaya et al. 1999). The profile shows variability of all its parameters, but our database is not sufficient to understand a regular character of the variability. Figure 4 presents histograms of velocities corresponding to the gravity centre of the emission profile $\left(V_{c}\right)$, to its blue edge $\left(V_{b}\right)$ and to the red one $\left(V_{r}\right)$. The distributions of these parameters show that, statistically, the emission He I profile in the spectrum of HD 190073 is slightly blueshifted. The mean values of $V_{b}, V_{c}$ and $V_{r}$ are correspondingly $-240,-16$ and $+180 \mathrm{~km} \mathrm{~s}^{-1}$. As one can see in Fig. 4 (right upper panel), a notable correlation $(R=0.72 \pm$ 0.12 ) exists between $V_{b}$ and $V_{r}$. It means that the line profile conserves its general type but oscillates in width. No relationship has been found between these oscillations and variations of other spectral parameters of the object. A similar behaviour of the He I $\lambda 5876 \AA$ line profile is also observed for the Herbig Ae star HD 36112 (Beskrovnaya et al. 1999).

\subsection{The NaID doublet}

The line profiles of the NaID doublet in the spectrum of HD 190073, shown in Fig. 5, are also very similar to those observed in the Herbig A8e star HD 36112 (Beskrovnaya et al. 1999). The lines show emission P Cyg II-type profiles which, in contrast to the Balmer lines, are seen on all the observing dates. Additionally, two narrow deep absorptions are present against the emission background, the first is centered at $-1.5 \pm 0.2 \mathrm{~km} \mathrm{~s}^{-1}$ and the second at $-17.7 \pm 0.2 \mathrm{~km} \mathrm{~s}^{-1}$, during the entire period of observations. Intensities of all the profile components are variable in time and do not correlate with other spectral variations observed in HD 190073. 

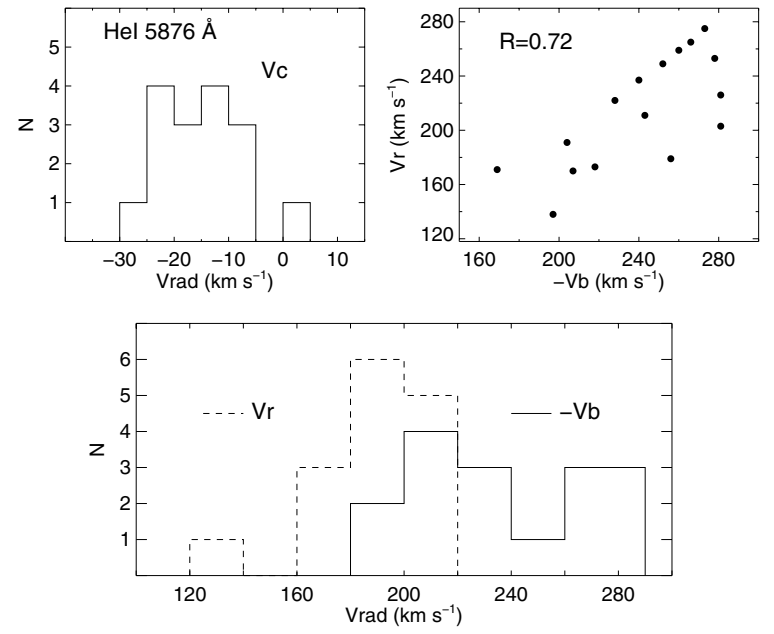

Fig. 4. Statistical characteristics of the He I $\lambda 5876 \AA$ line profile in the spectrum of HD 190073. Left upper panel: histogram of the centre gravity velocity $\left(V_{\mathrm{c}}\right)$ of the emission profile. Right upper panel: correlation between radial velocities corresponding to the blue edge $\left(V_{b}\right)$ and the red edge $\left(V_{r}\right)$ of the emission profile. Bottom panel: distribution of parameters $V_{b}$ and $V_{r}$.

It is remarkable that the blue edge of the full profile extends up to $-400 \mathrm{~km} \mathrm{~s}^{-1}$ whereas the extension of the red edge does not exceed $+200 \mathrm{~km} \mathrm{~s}^{-1}$. This effect is often seen in other HAEBEs (Pogodin 1997; Beskrovnaya et al. 1999).

\subsection{The multi-component $\mathrm{Ca} I I \mathrm{H}$ and $\mathrm{K}$ lines}

The échelle spectra of the object allowed us to investigate the most unusual spectral feature of HD 190073 - the complex structure of its $\mathrm{Ca}$ II (H and $\mathrm{K}$ ) profiles.

Figure 6 illustrates the mean profiles of the $\mathrm{K}$ and $\mathrm{H}$ lines in comparison with the synthetic spectrum calculated using the photospheric Kurucz's model: $T_{\text {eff }}=9300 \mathrm{~K}, \log g=3.5$, $v \sin i=12 \mathrm{~km} \mathrm{~s}^{-1}$, as we have already used for computing the $\mathrm{H} \gamma$ and $\mathrm{H} \delta$ profiles. The synthetic spectrum allows us to normalize the spectra and identify photospheric lines contributing to the fine multi-component structure of the profiles. We have found that the features centered at -123 and $-256 \mathrm{~km} \mathrm{~s}^{-1}$ are really due to the photospheric Ti II $\lambda 3932.0$ and $\mathrm{Fe} \mathrm{I} \lambda 3930.3 \AA$ lines.

Table 4 presents information on radial velocities of stable CS blueshifted absorptions forming the full profile of each of the $\mathrm{K}$ and $\mathrm{H}$ components of the violet $\mathrm{Ca}$ II doublet separately for the 1994-1997 and 1999-2000 observing seasons. Superpositions of local features ranked among the groups A, $\mathrm{B}$ and $\mathrm{C}$ were observed previously in a form of three main absorption components (see Sect. 1). But, as one can see in Figs. 6 and 7, in the 90's the single absorption B centered at $-180 \mathrm{~km} \mathrm{~s}^{-1}$ broke down into two separate components at -170 and $-200 \mathrm{~km} \mathrm{~s}^{-1}$. Taking into account that the accuracy of the velocity measurements was $\pm 5-10 \mathrm{~km} \mathrm{~s}^{-1}$ for the photographic data and $\pm 1-2 \mathrm{~km} \mathrm{~s}^{-1}$ for our spectroscopy with modern spectrographs equipped with CCD detectors, we can state that a real change of the global profile structure took place between the 80's and the 90's.
A detailed analysis of the local blueshifted absorptions shows that all of them were present in the spectrum of HD 190073 as early as in the 1940s-1970s (see Table 4). But, although the global profile structure remained stable for decades, the intensities and velocities of the local features were variable during all the observing seasons. In particular, the typical amplitude of positional variability was $10-20 \mathrm{~km} \mathrm{~s}^{-1}$ for different components on a timescale of several years. Our observational data also confirm the velocity change of some local components. So, the feature seen in 1994-1997 at -202 $\mathrm{km} \mathrm{s}^{-1}$ was observed at $-194 \mathrm{~km} \mathrm{~s}^{-1}$ in 1999-2000. The same positional variations of two other features were: from -172 to $-166 \mathrm{~km} \mathrm{~s}^{-1}$ and from -331 to $-323 \mathrm{~km} \mathrm{~s}^{-1}$. The feature at $-272 \mathrm{~km} \mathrm{~s}^{-1}$ was observed only in 1994-1997 and those at -284 and $-340 \mathrm{~km} \mathrm{~s}^{-1}$ - only in 1999-2000. These variations are illustrated in Table 4 and Fig. 6 (bottom panel). The intensities of the local absorption features show variations on a shorter timescale of several months (see Fig. 7).

Besides the division of the $\mathrm{B}$ component at $-180 \mathrm{~km} \mathrm{~s}^{-1}$ into two isolated absorptions at -200 and $-170 \mathrm{~km} \mathrm{~s}^{-1}$, which is discussed in this paper, a large-scale profile transformation likely took place between the 1950s and 1970s when the depth of the B absorption became significantly shallower than the $\mathrm{C}$ absorption (Surdej \& Swings 1976a, Fig. 4).

Thus the local components of the Ca II lines in the spectrum of HD 190073 demonstrate variability in intensity as well as in velocity position on timescales from months to decades.

As a result, we do not feel sure that the 2:1 ratio is a significant property of radial velocities of local components in the spectrum of HD 190073 (Scargle 1973; Tifft 1977). Our data are also not sufficient to confirm or rule out the correlation between the PCyg-structure of the $\mathrm{H} \gamma$ and $\mathrm{H} \delta$ lines as declared earlier by Surdej \& Swings (1976a,b, 1977). The profiles of the Ca II doublet obtained on dates when the PCyg-structure of Balmer lines was present or absent demonstrate no obvious characteristics which could be connected with one or other type of the Balmer line profiles (Fig. 6, bottom panel).

\subsection{CS emission lines and the shell-type spectrum}

The échelle spectral data also allow us to investigate the very rich line spectrum of HD 190073. We identified several hundreds of lines, which can be divided into two main groups. The first group includes emission lines with profiles with a single triangle-like peak overlapped by a central narrow absorption core at approximately zero radial velocity $+0.3 \pm 0.5 \mathrm{~km} \mathrm{~s}^{-1}$. The profiles are not symmetric, all of them possess a notable depression on the blue side. As a result, the bisector velocity $V_{\text {bis }}$ for the $0.5 F_{\max }$ level is significantly positive $\left(+6 \pm 2 \mathrm{~km} \mathrm{~s}^{-1}\right)$. The velocity limits of the emission are in the range $\pm 120-180 \mathrm{~km} \mathrm{~s}^{-1}$ for all lines with $F_{\max }$ from 1.1 to 1.3 units of the continuum. The widths of their central absorption cores are approximatelly the same $\left( \pm 15-20 \mathrm{~km} \mathrm{~s}^{-1}\right)$. This profile type is observed mainly in ionized metals (Fe II, Ti II) and, also, in such elements as Si II, Cr II, Mg I. The strongest of them are Fe II (42) lines at 4924, 5018 and $5169 \AA$ (Figs. 8 and 9). The asymmetry of these Fe II line profiles is even more 


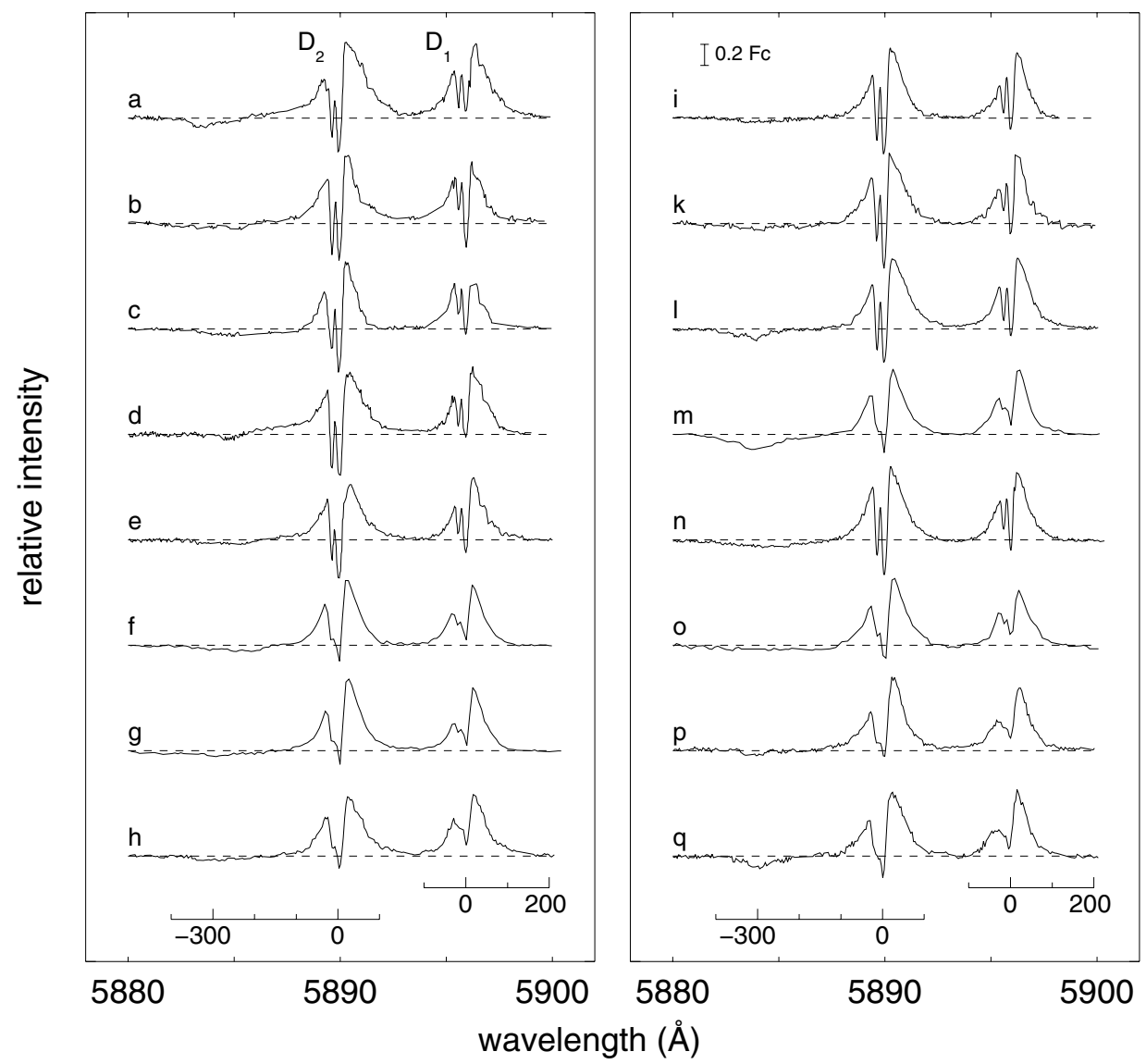

Fig. 5. Profiles of the NaID doublet observed on different dates marked by the same letter designations as in Fig. 1. The velocity scale with respect to the star is given separately for the $\mathrm{D}_{1}$ and $\mathrm{D}_{2}$ lines.

evident $\left(V_{\text {bis }}\right.$ for the $0.5 F_{\max }$ level is $+10 \pm 1 \mathrm{~km} \mathrm{~s}^{-1}$ ). Two more intense lines at $\lambda 5018 \AA$ and $\lambda 5169 \AA$ demonstrate also the PCyg profile structure with the velocity of the blue edge being $-400 \mathrm{~km} \mathrm{~s}^{-1}$, while at the same time the red edge velocity is only $+200 \mathrm{~km} \mathrm{~s}^{-1}$ (Fig. 9).

In addition to the emission lines, numerous narrow purely absorption shell-like lines of Fe I, Fe II, Sc II, Al I, Ti I, etc. are also seen in the spectrum. The blue wing of the Fe II (42) $\lambda 4923.9 \AA$ line is partially overlapped by two blends Fe I+Ni I $(\lambda 4919.0 \AA)$ and Fe I+Co I+Co III ( $\lambda 4920.5 \AA)$. There is no reason to suppose that this line possesses a multicomponent structure, like the one observed in the violet Ca II doublet, as suggested by Ringuelet \& Sahade (1984). As illustrated in Fig. 9, the shell-like profiles closely correspond to the synthetic photospheric spectrum calculated for the Kurucz' model used for the definition of the photospheric background near the ultraviolet $\mathrm{Ca}$ II $\mathrm{K}$ and $\mathrm{H}$ lines.

This raises the question of whether all narrow absorption lines, including the cores of emission lines, are really of a CS nature, or instead originate in a slow-rotating photosphere. If the latter is the case, the profiles of these photospheric lines have to be constant in time. Figure 9 also contains the residual spectra in the regions $\lambda \lambda 4460-4500 \AA$ and $\lambda \lambda 5160-5200 \AA$ constructed for different dates relative to the spectrum obtained on June 23, 1999. These spectral bands contain a number of emission lines of Fe II, Mg II and Ti II as well as more than twenty shell-like lines of Fe II, Mg II, Ti II, Fe I, Mg I. Only emission lines are variable on a timescale of months, whereas all the shell-like absorptions as well as the central cores of emission lines are seemingly constant. Moreover, the profiles of the absorption lines are in good agreement with the ones predicted by the chosen theoretical model. We suppose that all of them are formed in the stellar atmosphere.

\subsection{The UV lines}

The $\mathrm{Mg}$ II $\mathrm{k}$ and $\mathrm{h}$ lines of HD 190073 at $2796.35 \AA$ and $2803.53 \AA$, respectively, are shown in Fig. 10. These lines have P Cyg profiles of type IV (Beals 1951), indicating the presence of wind, and are very similar to the one observed for AB Aur (Praderie et al. 1986). The blue-shifted absorption part of the profile is totally saturated and its blue wing velocity is equal to or larger than the one observed in the other atomic species $(\mathrm{H} \alpha$, $\mathrm{NaID}$, and $\mathrm{Ca}$ II H, K).

Inspection of the Mg II lines displayed in Fig. 10 reveals that the blueshifted absorption part of the $\mathrm{Mg}$ II line profile presents variable terminal velocity and that the redshifted emission component varies in intensity. A similar behaviour has been found for $\mathrm{AB}$ Aur, which presents systematic terminal velocity variations, possibly related to the stellar rotation period (Praderie et al. 1986). 
Table 4. Radial velocities of the blueshifted CS components of the CaII Hand K lines observed in the spectrum of HD 190073 in 1994-1997 (OHP data) and 1999-2000 (ESO data). The feature at $0-3 \mathrm{~km} \mathrm{~s}^{-1}$ is likely to be a blend of the photosferic and the IS components (see Fig 6).

\begin{tabular}{cccc}
\hline \hline $\begin{array}{c}\text { Groups of } \\
\text { CS components }\end{array}$ & $\begin{array}{c}\text { OHP } \\
(1994-1997)\end{array}$ & $\begin{array}{c}\text { ESO } \\
(1999-2000)\end{array}$ & $\begin{array}{c}\text { Previous data } \\
(1943-1974)\end{array}$ \\
\hline A & $0 \pm 1$ & $+3 \pm 2$ & $+5--5$ \\
& $-17 \pm 1$ & $-15 \pm 2$ & $-12--15$ \\
B & $-145 \pm 1$ & $-146 \pm 1$ & $-143--155$ \\
& $-172 \pm 1$ & $-166 \pm 1$ & $-168--188$ \\
& $-202 \pm 1$ & $-194 \pm 1$ & $-198--212$ \\
& $-225 \pm 2$ & $-226 \pm 2$ & $-227--237$ \\
& $-236 \pm 1$ & $-238 \pm 3$ & $-238--243$ \\
C & $-272 \pm 2$ & - & $-265--274$ \\
& - & $-284 \pm 1$ & \\
& $-294 \pm 1$ & $-295 \pm 1$ & different sets \\
& $-305 \pm 3$ & $-304 \pm 1$ & of local \\
& $-331 \pm 1$ & $-323 \pm 2$ & features between \\
& $-354 \pm 2$ & $-358 \pm 3$ & -281 and -363 \\
& & & \\
& $-384 \pm 3$ & $-383 \pm 2$ & $-388--400$ \\
\hline
\end{tabular}

The velocities are given in $\mathrm{km} \mathrm{s}^{-1}$, their errors $( \pm \sigma)$ are derived as deviations of measurements obtained on different dates from the mean value. The previous data (1943-1974) presented in the last column were collected by Surdej \& Swings (1976a). The features at -123 and $-256 \mathrm{~km} \mathrm{~s}^{-1}$ are actually due to the Fe I photospheric lines and are not included in the table. The feature at $0-3 \mathrm{~km} \mathrm{~s}^{-1}$ is likely to be of IS origin.

The ultraviolet Fe II spectrum of HD 190073 is rich in lines. Figure 11 illustrates two spectral regions which are dominated by numerous Fe II lines. The upper panel displays the region $\lambda \lambda 2550-2640 \AA$ which contains many lines of the UV multiplets 1 and 64 , and the middle panel displays the region $\lambda \lambda 2720-2770 \AA$ containing lines of the UV multiplets 62 and 63 , as obtained on June 18, 1993. The vertical lines indicate the location of the Fe II line transitions. When not too blended, all Fe II lines show an undisplaced narrow component (probably of interstellar origin) and a wide blueshifted associated component. Enlarged diagrams for the doublet $\lambda 2599.15 \AA$ and $\lambda 2600.17 \AA$ of Fe II (1), and for the line $\lambda 2756.55 \AA$ of Fe II (62) are given in the bottom panels. In the lower part of each of these panels we indicate the corresponding position of the identified violet Ca II components (see Table 4).

The remarkable correspondence indicates that both the UV Fe II and the violet Ca II lines are probably formed in the same volume of space.

For Fe II $\lambda 2756.55 \AA$ (Fig. 9, right bottom panel), components " $A$ " (interstellar?) are absent - they barely seen only in the spectrum of October 11, 1980. Another visible variation can be noticed in the profile observed on June 18, 1993 when the shortward component $\left(\sim-400 \mathrm{~km} \mathrm{~s}^{-1}\right)$, observed in the other two nights, faded as did the redward part of the B components.

\section{Discussion}

The results of our spectroscopic investigation of HD 190073 show that this early-type object with emission lines demonstrates properties making it typical as well as unusual among other Herbig B8e-A2e stars with signs of strong stellar wind (see Table 1). These properties can be summarized as follows:

1. All emission lines observed in the object's spectrum originate in a non-stable wind. The optically thick lines (the Balmer lines, the red $\mathrm{Na}$ I and blue $\mathrm{Ca}$ II doublets and the Fe II (42) lines) show variable PCyg-type profiles. The profiles demonstrate pronounced asymmetry with the blue part being much more extended. The blue edge of their PCyg-absorption can be seen up to $-400 \mathrm{~km} \mathrm{~s}^{-1}$ while the red edge of the emission wing is limited to about $+200-250 \mathrm{~km} \mathrm{~s}^{-1}$.

2. The same profile type is also observed in the UV resonance $\mathrm{Mg}$ II ( $\mathrm{k}$ and $\mathrm{h}$ ) doublet and in a number of Fe II lines in the 2500-2800 A region. Similar to strong visual lines with PCyg-type profiles, the profiles of the UV resonance lines demonstrate significant time variability. A number of local features seen in the $\mathrm{Ca}$ II ( $\mathrm{H}$ and $\mathrm{K}$ ) profiles also seem to be observed in the UV lines. As a whole, the local absorptions in the UV profiles are much deeper and look like a single wide blend of almost zero intensity (Fig. 11). The same $\mathrm{Mg}$ II profile type is typical for other Herbig Ae stars with signs of strong stellar wind (for example, AB Aur, Imhoff 1994). We cannot exclude that the Ca II ( $\mathrm{H}$ and $\mathrm{K}$ ) profiles of all Herbig Ae stars would possess a similar complex structure as HD 190073, but larger values of $v \sin i$ could smooth out this structure in other objects. Detection of weak blueshifted absorption components of the Ca II K line in the spectrum of $\mathrm{AB}$ Aur has been reported by Catala et al. (1986b), and by broadening the observed Ca II K profile of HD 190073 one can obtain a profile that resembles the one observed for HD 250550 (Catala et al. 1991).

3. The UV line profiles in the spectrum of HD 190073 also demonstrate different extension of the blue edge $\left(-500 \mathrm{~km} \mathrm{~s}^{-1}\right)$ and the red edge $\left(+200 \mathrm{~km} \mathrm{~s}^{-1}\right)$. This phenomenon is likely to be a result of the fact that dense matter concentrated near the equatorial plane screens the receding zone of the stellar wind. We propose that this matter is concentrated in an optically thick CS disk, as is observed around a number of Ae/Be Herbig stars where it is responsible for their far IR dust emission. Other emission lines with a single profile type originate also in the stellar wind. But the wind is not so opaque in these lines and is displayed only in the form of a blue depression. The narrow photospheric lines overlap the central part of the profiles.

4. The emission Balmer decrement of HD 190073 is unusually gently sloped (Sect. 3.1). Taking into account that the emission $\mathrm{H} \alpha$ line in the spectrum of the object is of medium intensity ( $W=40 \AA$ ), this fact provides evidence that the envelope around the star is rather more dense and compact than for other HAEBE stars (Pogodin 1986). It would be interesting to investigate this fact in forthcoming studies of this object. 

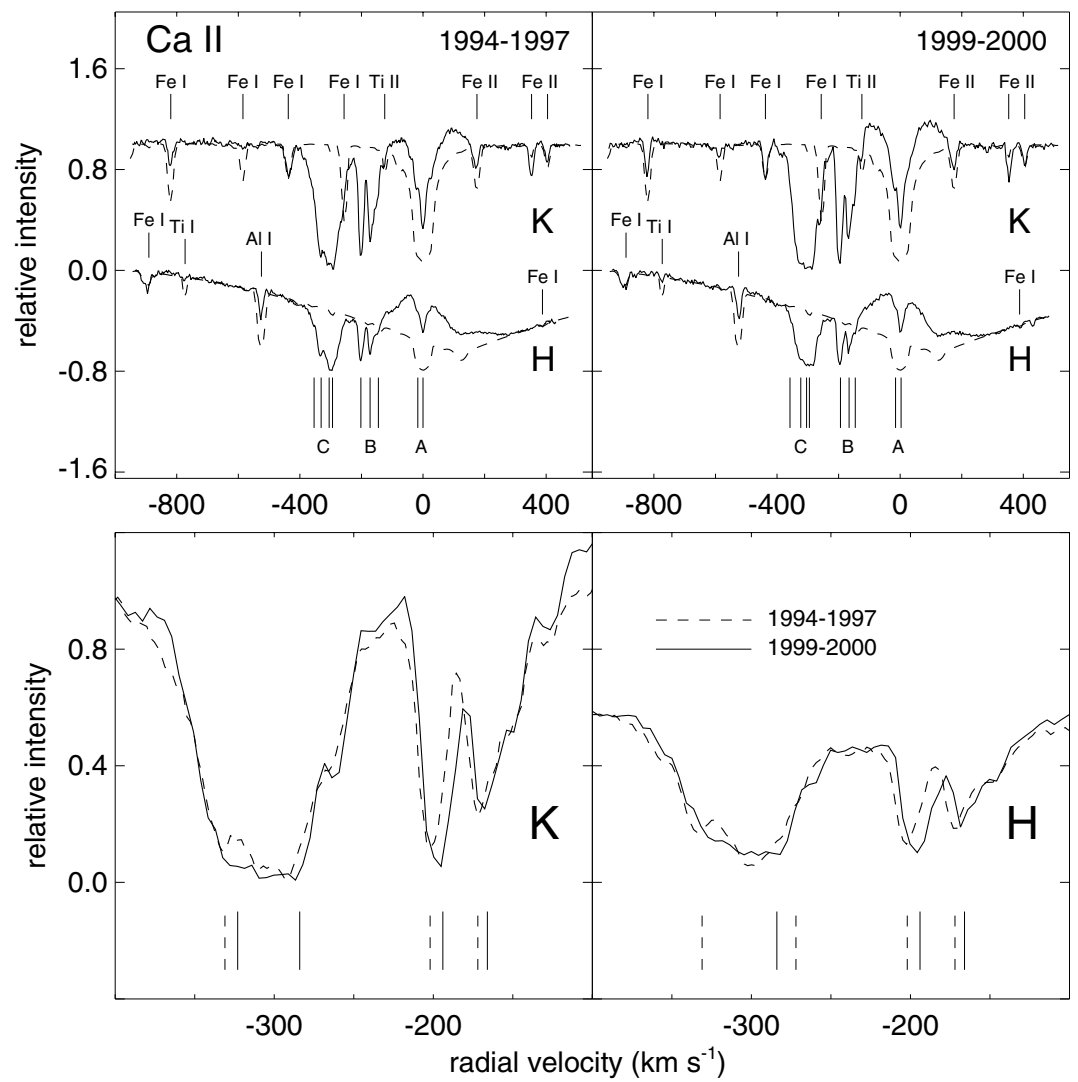

Fig. 6. The mean multi-component profiles of the Ca II K and H doublet observed in the spectrum of HD 190073 in $1994-1997$ (left upper panel) and in 1999-2000 (right upper panel). A synthetic theoretical spectrum is given for comparison (dashed lines). The positions of photospheric lines and three main groups of CS Ca II components A, B and C are shown using thin vertical lines. Differences of the fine K and H profile structure observed in 1994-1997 and 1999-2000 are illustrated in the lower panels. The vertical lines indicate features observed only in 1994-1997 (dashed lines) and in 1999-2000 (solid lines).

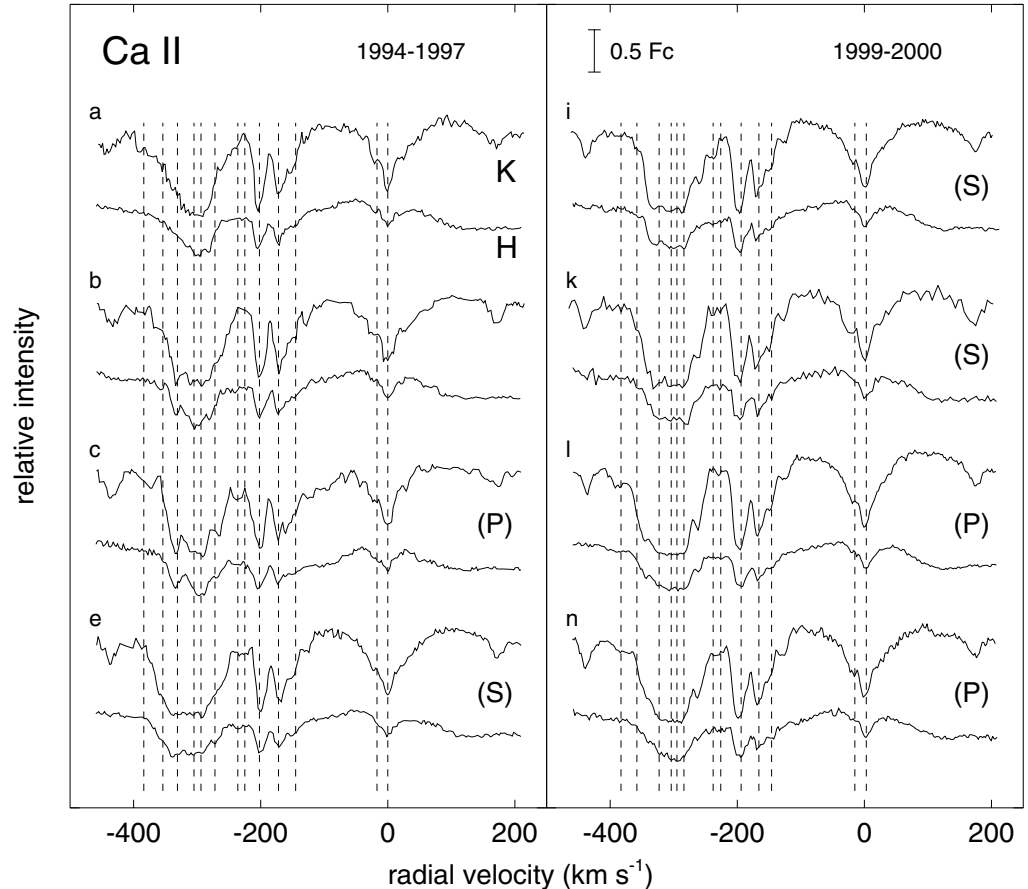

Fig. 7. Individual profiles of the $\mathrm{Ca}$ II $\mathrm{K}$ and $\mathrm{H}$ lines obtained on different dates marked by the same letters as in Fig. 1. The velocity positions of CS Ca II components are indicated by thin vertical dashed lines (see Table 4). The dates, when the $\mathrm{H} \gamma$ and $\mathrm{H} \delta$ in the spectrum of HD 190073 demonstrated the PCyg-type or single emission profiles are denoted by symbols $(\mathrm{P})$ and $(\mathrm{S})$ respectively. 

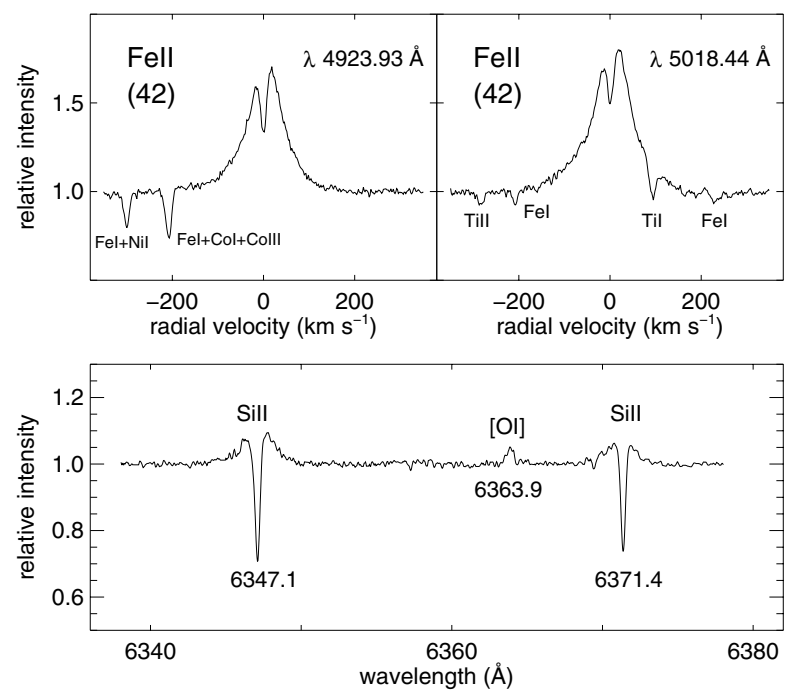

Fig. 8. Typical emission and shell-type line profiles observed in the spectrum of HD 190073.

5. The unique multi-component profiles of the violet Ca II doublet demonstrate a complex picture of variability displayed on timescales from months to decades. This variability is in rather poor agreement with the traditional interpretation of the phenomenon as the result of the radiative forces acting selectively on the outflowing gas and stimulating a stratification of the stellar wind with distance from the star. The mechanism described by Scargle (1973) and Tifft (1977) as well as the interpretation proposed by Surdej \& Swings (1976b) require: a) the absence of positional variability of the local absorption components; and b) the prevalence of the phenomenon among all early-type objects with CS envelopes. Neither of these two conditions are met. The fine structure of the Ca II profiles is far from being stable and HD 190073 is, so far, the only HAEBE star displaying such complex shapes of the Ca II profiles.

A small value of $v \sin i$ determined for HD 190073 (see Table 1) offers evidence that: a) the object has a "pole-on" orientation; or b) its orientation is intermediate, but the rotation velocity of the star is small. Let us consider these two possibilities to appreciate the general properties of the CS environment of HD 190073, and how unusual this star is among others HAEBEs.

Assuming that the object is a "pole-on" star we run into a problem. If it were the case, HD 190073 would be required to possess a polar stellar wind. According to the majority of current physical scenarios, the wind of HAEBEs is more likely to be generated at lower stellar latitudes than at higher ones. A number of recent theoretical articles are devoted to the formation of polar outflowing jets from young low-mass stellar objects near the star (e.g., Turner et al. 1999; Goodson et al. 1999), but it is necessary to justify, on the basis of MHD calculations, that the appearance of a strong stellar wind near the polar region of a HAEBE is not improbable. Spectroscopic studies of some HAEBEs with "pole-on" orientation (see, for
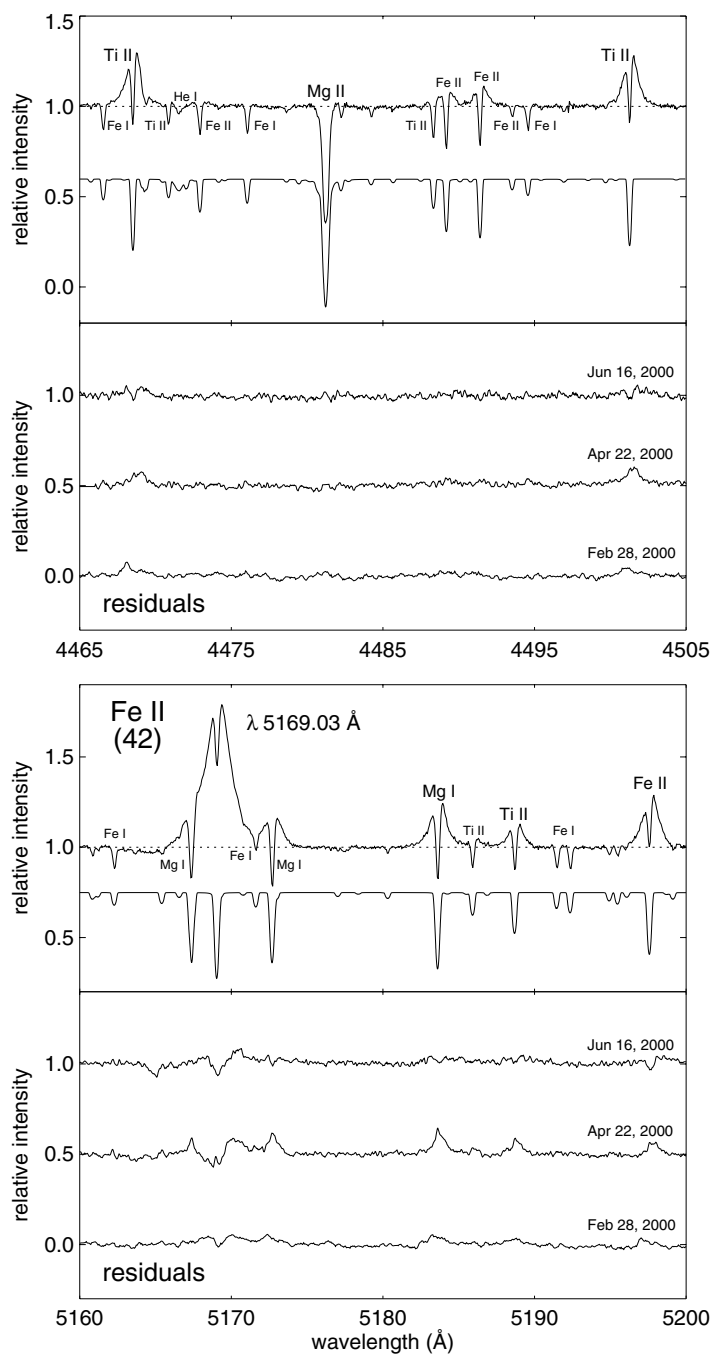

Fig. 9. The mean spectrum of HD 190073 in the regions $\lambda \lambda 4460-4500 \AA$ and $\lambda \lambda 5160-5200 \AA$ created by averaging four spectra obtained at the ESO on dates: i) June 23, 1999; k) February 28, 2000; 1) April 22, 2000; and n) June 16, 2000. The synthetic theoretical spectra are given for comparison below the observational spectra. The residual spectra calculated relative to the first of them for these dates are also presented.

example, HD 179218, Kozlova 2004) showed that a polar wind is not an attribute of these objects.

The assumption that HD 190073 is oriented relative to the observer at an intermediate angle and exhibits an axially symmetric global magnetic field was made by Cuttela \& Ringuelet (1990). In spite of the fact that the magnetic properties of HAEBEs with CS disks remain to be explored, we reason that the existence of a field with topology analogous to that recognized for low-mass T Tau-type stars can be realistic (see the series of models by Camenzind 1990; Königl 1991; Hartmann et al. 1994; Shu et al. 1994, etc.).

In contrast to the traditional interpretation of the peculiar profile of the blue $\mathrm{Ca}$ II doublet, based on a radial stratification of the outflowing CS gas (see Sect. 3.4), we suppose that the hypothetical axially symmetric magnetic field of a specific configuration can result in a quasi-stable latitudinal stratification of 


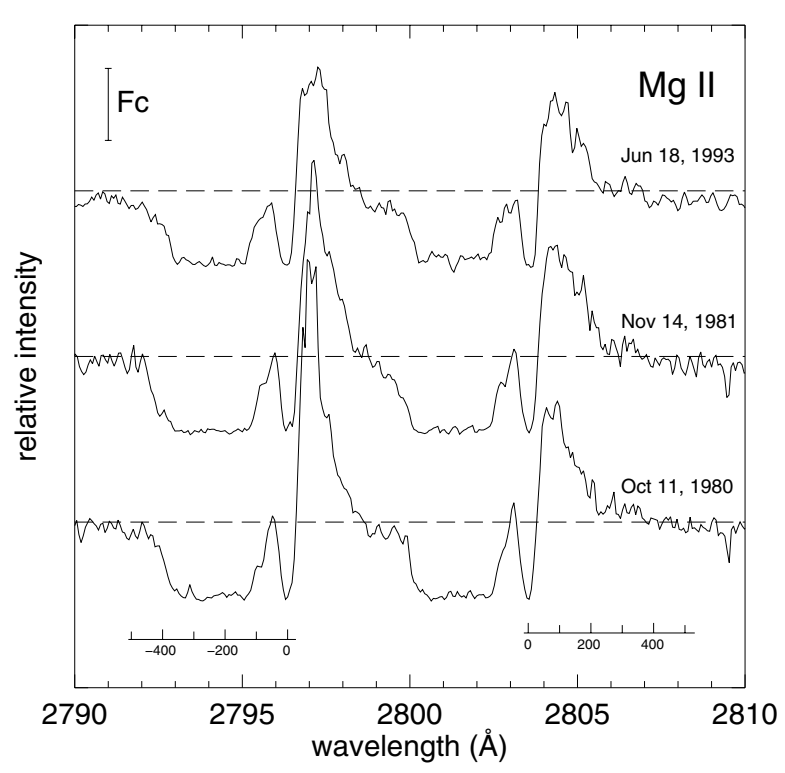

Fig. 10. Mg II $\mathrm{k}$ and $\mathrm{h}$ line profiles for HD 190073. The velocity scale with respect to the star is given separately for each line of the doublet.

the wind zone and stimulate the formation of multi-component $\mathrm{Ca}$ II $\mathrm{H}$ and $\mathrm{K}$ line profiles.

This assumption has to be confirmed by future measurements of the magnetic field of HD 190073 using modern instruments separately for lines of different elements as well as by MHD calculations of a possible magnetic configuration near stars such as HD 190073 as a result of its interaction with the equatorial CS disk.

\section{Conclusions}

The results of our cooperative spectroscopic programme have shown that the early-type emission line object HD 190073 displays properties common with other HAEBEs as well as peculiarities making the object unusual for typical Herbig stars of similar spectral classes and the P Cyg profiles of spectral lines.

Among the common characteristics which have been established in the framework of our observations:

1. Large-scale variability of the Balmer line profiles from the PCyg-type to a single peak.

2. A noticeable asymmetry of P Cyg-type profiles of CS lines with a dominating blue component as an additional indicator of an optically thick equatorial disk.

In addition, a number of distinctive features of HD 190073 have been revealed:

1. An anomalously gently-sloped emission Balmer decrement as an indicator of enhanced density of the CS emission gas.

2. During two consecutive observing nights we found no signs of the short-term variability of the $\mathrm{H} \alpha$ profile in the spectrum of HD190073. Such variability is typical of HAEBEs.

3. A slightly blueshifted, purely emission profile of the He I $\lambda 5876 \AA$ line without any signs of the red absorption

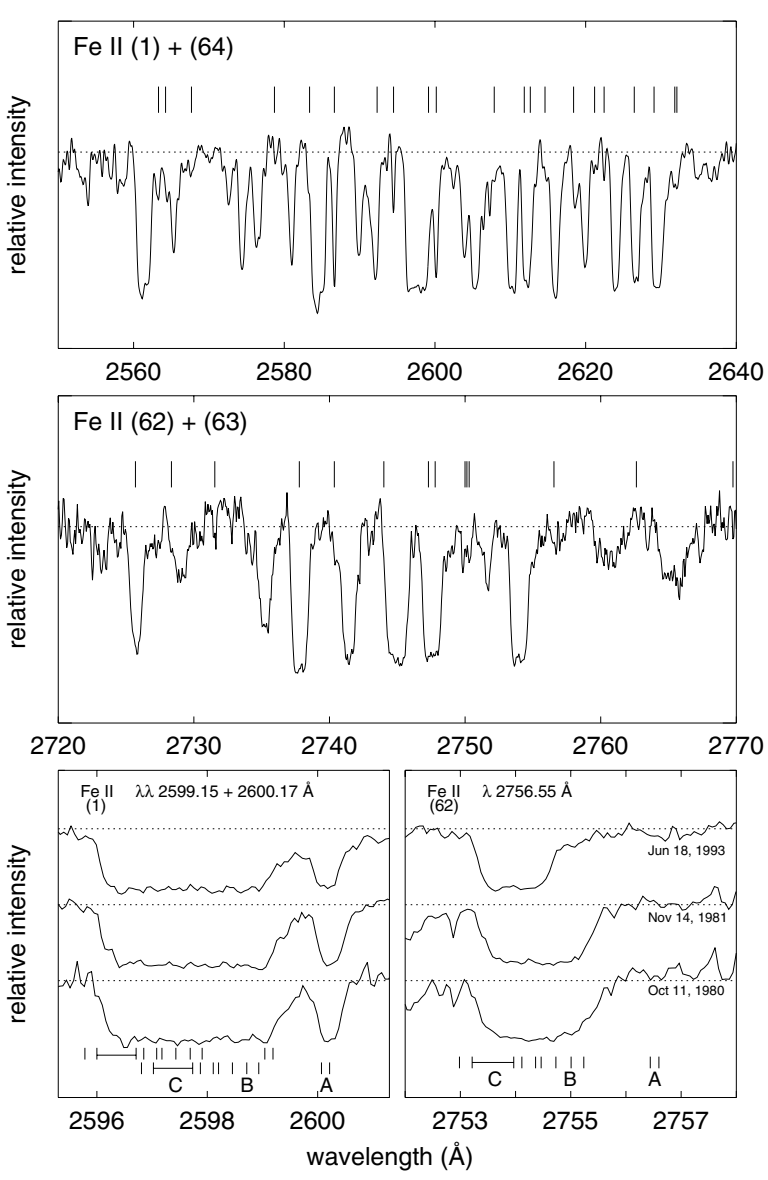

Fig. 11. The ultraviolet Fe II profiles in HD 190073: multiplets 1, and 64 (upper panel), and multiplets 62, and 63 (middle panel) observed on June 18, 1993 (LWP25762). The vertical lines indicate the location of the Fe II line transitions. Details of the doublet $\lambda 2599.15 \AA$ and $\lambda 2600.17 \AA$ (left bottom panel) and of the line $\lambda 2756.55 \AA$ (right bottom panel) are given for the high-resolution spectra. In both panels we indicate the position of the identified violet Ca II blueshifted CS components (see Table 4). For the left bottom panel both sets of components are marked, one for each line of the Fe II (1) doublet.

component typical for spectra of HAEBEs. Only the Herbig Ae star HD 36112 exhibits a similar profile for this line.

4. The well-known multi-component structure of Ca II (K and $\mathrm{H}$ ) profiles turns out to be not stable in time but demonstrates a complex variability on timescales from months to decades. This fact is not in agreement with the previous interpretation of the phenomenon as a result of a density stratification of the outflowing gas due to selectively acting radiative forces.

We believe that similar complex structure of the blue absorption component may be present not only in the Ca II ( $\mathrm{K}$ and $\mathrm{H})$ lines of HD 190073, but also in all resonance UV lines of others Herbig Ae stars, however, factors such the large optical thickness of the UV lines and large $v \sin i$ for the main part of the HAEBEs smooth out the components of these structures. This is in agreement with the results obtained by Catala et al. (1986a), who observed that the Ca II K profiles of a sample of known Herbig Ae stars demonstrate wide 
blue absorption components with local features; however, the shape and positions of these features are different for different objects.

We should like to emphasize the importance of future magnetometric observations to develop a true model of the CS environment around HD 190073 and to understand the peculiar properties of this object.

Acknowledgements. The authors gratefully acknowledge the editor, Dr. S. N. Shore, and the anonymous referee for their valuable comments that have improved the paper. The Brazilian authors acknowledge the financial support by CNPq. This research has made use of IUE observations retrived from the INES archives.

\section{References}

Allen, D. A., \& Swings, J. P. 1976, A\&A, 47, 293

Babcock, H. W. 1958, ApJS, 3, 141

Beals, C. S. 1951, Publications of the Dominion Astrophysical Observatory Victoria, 9, 1

Beskrovnaya, N. G., Pogodin, M. A., Miroshnichenko, A. S., et al. 1999, A\&A, 343, 163

Beskrovnaya, N. G., Pogodin, M. A., Najdenov, I. D., \& Romanyuk, I. I. 1995, A\&A, 298, 585

Camenzind, M. 1990, Rev. Modern Astron., 3, 234

Catala, C., \& Kunasz, P. B. 1987, A\&A, 174, 158

Catala, C., Czarny, J., Felenbok, P., \& Praderie, F. 1986a, A\&A, 154, 103

Catala, C., Felenbok, P., Czarny, J., Talavera, A., \& Boesgaard, A. M. 1986b, ApJ, 308, 791

Catala, C., Czarny, J., Felenbok, P., Talavera, A., \& The, P. S. 1991, A\&A, 244, 166

Chen, P. S., Wang, X. H., \& He, J. H. 2000, Ap\&SS, 271, 259

Cidale, L., Zorec, J., \& Morrell, N. 2000, in IAU Colloq. 175, The Be

Phenomenon in Early-Type Stars, ASP Conf. Ser., 214, 87

Corporon, P., \& Lagrange, A.-M. 1999, A\&AS, 136, 429

Cuttela, M., \& Ringuelet, A. E. 1990, MNRAS, 246, 20 de Winter, D., van den Ancker, M. E., Maira, A., et al. 2001, A\&A, 380, 609

Deul, E. R., \& van Genderen, A. M. 1983, A\&A, 118, 289

Glagolevskij, Y. V., \& Chountonov, G. A. 1998, Bull. Special Astrophys. Obs., 45, 105

Goodson, A. P., Böhm, K., \& Winglee, R. M. 1999, ApJ, 524, 142

Grady, C. A., Perez, M. R., Talavera, A., et al. 1996, A\&AS, 120, 157

Hartmann, L., Hewett, R., \& Calvet, N. 1994, ApJ, 426, 669

Imhoff, C. L. 1994, in The Nature and Evolutionary Status of Herbig Ae/Be Stars, ASP Conf. Ser., 62, 107

Königl, A. 1991, ApJ, 370, L39

Kozlova, O. V. 2004, Astrophys., 47, 287

Lamers, H. J. G. L. M., \& Waters, L. B. F. M. 1984, A\&A, 136, 37

Low, B. C., \& Tsinganos, K. 1986, ApJ, 302, 163

Malfait, K., Bogaert, E., \& Waelkens, C. 1998, A\&A, 331, 211

Merrill, P. W. 1933, ApJ, 77, 51

Mora, A., Merín, B., Solano, E., et al. 2001, A\&A, 378, 116

Pogodin, M. A. 1985, AZh, 62, 918

Pogodin, M. A. 1986, Astrofiz., 24, 279

Pogodin, M. A. 1994, A\&A, 282, 141

Pogodin, M. A. 1997, A\&A, 317, 185

Praderie, F., Catala, C., Simon, T., \& Boesgaard, A. M. 1986, ApJ, 303, 311

Ringuelet, A. E., \& Sahade, J. 1984, The Messenger, 36, 23

Scargle, J. D. 1973, ApJ, 179, 705

Shu, F., Najita, J., Ostriker, E., et al. 1994, ApJ, 429, 781

Sitko, M. L. 1981, ApJ, 247, 1024

Sitko, M. L., Meade, M. R., \& Savage, B. D. 1981, ApJ, 246, 161

Struve, O., \& Swings, P. 1942, ApJ, 96, 475

Surdej, J., \& Swings, J. P. 1976a, A\&A, 47, 113

Surdej, J., \& Swings, J. P. 1976b, A\&A, 47, 121

Surdej, J., \& Swings, J. P. 1977, A\&A, 54, 219

Swings, P., \& Struve, O. 1940, ApJ, 91, 546

Tifft, W. G. 1977, ApJ, 211, 377

Turner, N. J., Bodenheimer, P., \& Różyczka, M. 1999, ApJ, 524, 129 van den Ancker, M. E., de Winter, D., \& Tjin A Djie, H. R. E. 1998, A\&A, 330, 145

van Genderen, A. M. 1971, A\&A, 14, 48 